\title{
ANTROPOLOGÍA
}

\section{ANTROPOLOGÍA DE LOS CUIDADOS}

\section{ENFERMEDAD Y PADECIMIENTO: SIGNIFICADOS DEL ENFERMAR PARA LA PRÁCTICA DE LOS CUIDADOS}

\author{
Manuel Amezcua \\ Unidad de Ginecologia y Obstetricia \\ Hospital Universitario San Cecilio, Granada, España
}

\section{RESUMEN}

El autor valora, en este trabajo, la evolución de la antropología de los cuidados en España analizando el último lustro. En los últimos cinco años se ha producido un inesperado incremento del interés de las enfermeras por la relación entre la cultura y los cuidados.

Palabras clave: Antropologia, enfermeria.

\section{ANTHROPOLOGY OF CARES}

\section{SUMMARY}

In this article, the author values the evolution of the anthropology of cares in Spain analyzing the last lustrum. In the last five years an unexpected increase of the interest of the nurses by the relation between culture and cares.

Keywords: Anthropology, nursing.

En el último lustro estamos asistiendo en nuestro país a un interés creciente de las enfermeras por la relación entre la cultura y los cuidados (las Jornadas Internacionales sobre Cultura de los Cuidados, patrocinadas por la Universidad de Alicante, podrian considerarse muy bien la oficialización de una nueva enfermería enmarcada en el pensamiento hermenéutico). Las teorías de Leininger, probablemente la primera que realizó un verdadero esfuerzo por hermanar la antropología y la enfermería (Leininger, 1970) y de otras pensado- ras estadounidenses que le siguieron, han tardado casi una treintena de años en llegar a nuestro país, lo cual se entiende si consideramos la estrechez intelectual con la que se ha enseñado la enfermería en España en la segunda mitad de siglo. Tampoco la realidad social de nuestro país durante este tiempo, caracterizada por un etnocentrismo durante la dictadura franquista ("Spain is different", ise acuerdan?) y por una adscripción excesivamente radical a la globalidad en los últimos tiempos, ha favorecido el tomar conciencia de la importancia que tiene la consideración de la diversidad cultural a la hora de practicar cuidados de salud eficaces.

El interés creciente por la cultura de la salud entre nuestras colegas puede tener diversas causas:

- La necesidad de incorporar nuevos referentes teóricos en un momento de búsqueda incesante de identidad y de construcción disciplinar, ante los nuevos retos de crecimiento tanto en lo profesional como en lo académico.

- La necesidad de generar nuevas herramientas que nos permitan manejar adecuadamente los problemas de enfermería que se asocian a la diversidad cultural, favorecida en la última década por un notable aumento en nuestro país de la inmigración desde el continente africano, América latina y Europa oriental (Solas y col, 1995).

—Las posibilidades que los estudios de antropología (por ser una titulación de segundo 
ciclo) dan a las enfermeras para crecer académicamente allí donde su disciplina no le ofrece más que limitaciones.

Sea como fuere, este interés se ha manifestado en un nuevo discurso, que es posible compartan aún muy pocas enfermeras, en el que se mezclan:

-referentes filosóficos como la fenomenología, utilizada como nuevo paradigma para combatir o compensar el positivismo más seco en el que hemos sido educados la mayoría, debido a la medicalización tanto de los estudios como de la práctica profesional,

- con referentes teóricos como el transculturalismo o la etnoenfermería, que nos está sirviendo para descubrir nuevos espacios de cuidados (el trabajo con la comunidad, con las familias, con grupos étnicos, con marginados, etc) o para reorientar o redefinir nuestra aportación específica a problemas tradicionales de la salud (así aparecen nuevas ideas asociadas al cuidado, como el sufrimiento, lo que se ha dado en llamar el cuidado informal, etc), -y con referentes metodológicos como la investigación cualitativa (Amezcua y col, 2000), que nos está sirviendo para conocer la realidad descle otra perspectiva, la que tiene que ver con la interpretación que hace el propio sujeto en su contexto social (dimensión emic), para diferenciarla de nuestras interpretaciones como profesionales o como investigadores adoctrinados por nuestro propio saber científico (dimensión etic).

En cualquier caso, el maridaje entre enfermería y antropología (o entre cuidados y cultura) no se entendería sin una aproximación holística hacia el ser humano, si no entendiéramos que en el proceso de salud-enfermedad, no sólo debemos considerar los efectos materiales del deterioro biológico, sino que tanta importancia tienen los códigos de respuesta psico-social, que a su vez están

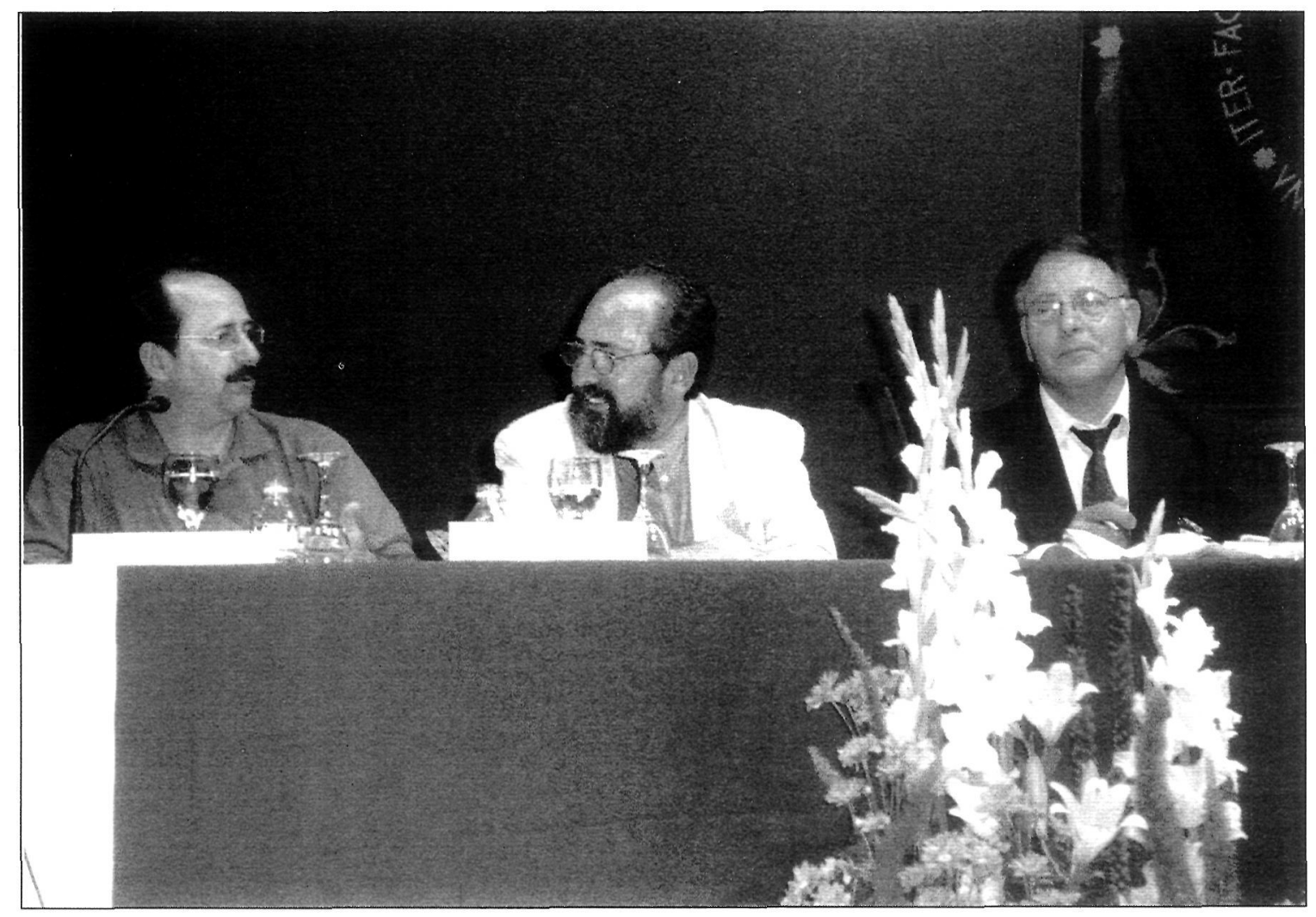


condicionados por los referentes culturales en los que las personas se hayan inmersas. O como diría Leininger en su concepto de cultura, del "conjunto de valores aprendidos y transmitidos; las creencias, normas de conducta y prácticas de un modo de vida de la gente en particular" (Leininger, 1978). Con todo ello podremos practicar unos cuidados culturalmente adaptados, que es lo que al fin y al cabo pretende el movimiento de la enfermería transcultural.

\section{El choque cultural}

La antropología nos ayuda, entre otras muchas cosas, a comprender los significados que el paciente y su familia otorgan a la enfermedad, nos adentra en su universo cultural, que no siempre, me atrevería a decir que casi nunca, coincide con el universo supuestamente docto en el que nos hayamos inmersos como profesionales. Y digo supuestamente porque la propia cultura científica nos debería dotar de la suficiente sensibilidad para comprender las realidades ajenas, cosa que casi nunca ocurre. Es más, me atrevería decir que por efecto del carácter excluyente de la ciencia, los profesionales de la salud (y mucho más los que más doctos se creen) nos comportamos como verdaderos analfabetos culturales ante el hecho diferencial.

¿Cuántas veces hemos escuchado afirmaciones tan rotundas como "iAquí el médico soy yo!", cuando un paciente pone en duda una terapia o sugiere otras posibilidades? Por lo general, en ese momento de choque profesional-paciente, es el paciente el que toma pronta conciencia de su posición desigual respecto al sistema médico hegemónico, de su relación de cautividad, de la condición de cuerpo-objeto con la que es requerido, y se calla, y otorga.

Pero ¿por qué se produce el choque cultural? Porque la salud y la enfermedad tienen significados en ocasiones muy distintos para el paciente que para el profesional, y por lo tanto incorporan valo- res, comportamientos y objetos diferentes. Cuando la enfermedad trasciende del espacio institucional médico y se sitúa en el contexto social y cultural del paciente a menudo varía su significado. Los significados de la enfermedad, que son fruto del consenso establecido por las particularidades de cada cultura (Fábrega, 1975:969), pueden ser tan diversos y contradictorios como los actores que intervienen en el padecimiento. Comprender la diversidad de significados supone la posibilidad de comprender al paciente y su familia en su ser único e irrepetible y de practicar unos cuidados culturalmente adaptados.

Pondré un ejemplo. Cuando un médico decide el ingreso del paciente en el hospital, éste es etiquetado a través de un diagnóstico, que determina la unidad donde será destinado (generalmente se identifica con un servicio médico). El diagnóstico le otorga la calidad de objeto (Josefa Pérez pasará a ser la histerectomía de la doce-dos) con un comportamiento esperado, que viene determinado en el protocolo de cuidados. Durante su estancia en el hospital le procuramos una cama en una habitación generalmente compartida, en la que tiene un aseo común y un pequeño armario como único espacio privado. Este esquema responde a la idea de habitabilidad que nosotros como profesionales tenemos para el enfermo-objeto.

Pero en la medida en que el proceso del paciente requiera una estancia más larga, la habitación se irá transformando para adaptarse a la idea de habitabilidad que tiene el paciente-persona, y que tenderá a recuperar aquellos objetos que son habituales en su mundo exterior, a reproducir en lo posible el ambiente cotidiano que le resulta confortable: un televisor o una radio portátiles, un teléfono móvil, una hamaca, revistas y periódicos, incluso se colocará junto al sofisticado cabecero de la cama una fotografía del patrón del pueblo, o varias estampas de los santos de mayor devoción. El aseo se convertirá en tendedero, el armario en 
una despensa, el pollo de la ventana, cuando no le dé el sol, en improvisado refrigerador, y de noche, cuando oficialmente han finalizado la mayoria de las rutinas, la habitación se trocará en un dormitorio comunitario donde se acuestan el doble de personas que camas hay.

En. nuestros hospitales y debido a la idea que tenemos de la familia y de su papel en el cuidado, se suele aceptar que haya una persona que acompañe al paciente durante el periodo de hospitalización. Este familiar-acompañente, que generalmente es la persona más cercana emocionalmente (esposo-esposa, hija, etc.) se presta a ello porque en realidad aspira a realizar en el hospital unos cuidados parecidos a los que realizaría de estar en casa. Pero con el tiempo termina adiestrándose en algunos cuidados básicos típicamente hospitalarios, como la higiene en cama, la vigilancia del suero o de las sondas, etc. Su implicación es tal en muchos casos que en determinadas unidades donde los pacientes tienen muy deteriorada su autonomía (oncológicos, infecciosos, etc) son un puente indispensable entre el paciente y la enfermera. Dicho de otra manera, si el hospital prescindiera de ellos no contaría con recursos humanos suficientes para atender adecuadamente a los pacientes.

Pero aunque el acompañante realiza unos cuidados consentidos, ejerce una función invisible para el sistema, lo cual produce contradicciones y por lo tanto choques. He visto cómo algunas enfermeras recriminan al acompañante cuando de noche permite que se le pase el suero del paciente (porque se ha quedado traspuesto), lo cual le obliga a coger de nuevo la vía ("para dormir más le valiera marcharse a su casa", vienen a decirle). Al día siguiente, una de las primeras medidas que las enfermeras toman es echar los acompañantes a la calle cuando los médicos van a pasar sala para que así no les molesten con preguntas fastidiosas. Los acompanantes tienen la obligación de cuidar a su paciente-persona, pero no tienen derecho a conocer lo que está ocurriendo con el enfermo-objeto salvo cuando lo dictan las normas.

Si un paciente ingresado está solo y precisa de muchos cuidados, alentamos a la lamilia a que se quede a acompañarle apelando a los beneficios que le puede procurar en el terreno emocional. Sin embargo, algunas enfermeras me han pedido que como responsable de la unidad recrimine a algunos acompañantes por utilizar para su higiene el aseo de la habitación, por ponerse un pijama de noche, por dejarse caer en la cama vacia de al lado, por tomar alimentos que el paciente desecha, o por albergar alimentos en la habitación. Nos preocupa. mucho la calidad de vida del enfermo-objeto porque sabemos que puede influir directamente sobre el mejoramiento de su proceso patológico, y así medimos de una manera obsesiva el balance hidroelectrolítico, la temperatura, el pulso, la respiración, la presión sanguínea, etc. (aunque la mayoría de las veces no se utilice para nada en concreto). Pero no parece preocuparnos en modo alguno la calidad de vida del acompañante del paciente-persona, ni nos preguntamos si el deterioro que puede sufrir al no poder satisfacer adecuadamente sus necesidades más básicas como persona (alimentación, higiene, reposo, etc.) puede debilitar su acción cuidadora.

Nuestro posicionamiento ambiguo o de choque hacia el acompañante es porque éste no tiene ninguna relación con el enfermo-objeto (con el hígado, el estómago, o el útero deteriorados), sino que pertenece al mundo del paciente-persona, donde el acompañamiento constituye un valor muy importante, al menos en nuestro contexto cultural. El acompañamiento no forma parte de la praxis que uno puede encontrar en los libros de patología médica, y sin embargo sería esperable encontrarlo como materia truncal en los manuales de enfermería (Juan Irigoyen, conocido sociólogo de la salud que padece una enfermedad crónica, 
mantenía en un reciente congreso de enfermería al que fue invitado que él, cuando acude al hospital, lo que en realidad espera de las enfermeras es que "le acompañen"). El "sentirse acompañado" es una necesidad expresada por el paciente-persona, no por el enfermo-objeto.

\section{Enfermedad y padecimiento}

Esta diferenciación que hago entre enfermoobjeto y paciente-persona no es más que otra estrategia para desafiar la concepción cartesiana de separación entre cuerpo y mente que ha caracterizado a nuestro sistema médico y aún le caracteriza en su desarrollo práctico. Siguiendo a Françoise Laplantine es posible y conveniente analizar los conflictos y convergencias entre los dos polos que están presentes en la práctica asistencial cotidiana y que necesariamente ha de condicionar la praxis enfermera:

-la "enfermedad en tercera persona", que hace mención al conocimiento objetivo de la biomedicina y de los valores que la sustentan,

-y la "enfermedad en primera persona", que se refiere al estudio de la subjetividad del enfermo a la hora de interpretar el proceso del enfermar, así como de la subjetividad del sanador o del cuidador (Laplantine, 1992:19).

Les propongo un escarceo lingüístico en este punto. Julio Casares recoge hasta 584 palabras en castellano que sirven para nombrar la enfermedad e ideas que le son afines (Casares, 1942:196-8). De ellas hay varias que son usadas cotidianamente como términos sinónimos porque hasta ahora no hemos precisado vertebrar el concepto de enfermedad: así hablamos de enfermedad y de enfermo, de padecimiento y de paciente, de mal o de malestar y de "estar malo" o "sentirme mal", de dolencia, de afección, de achaque, de molestia, de transtorno, de "estar delicado de salud" o de "tener mala salud", etc.

La lengua inglesa posee una triple terminología para designar la enfermedad, que los investiga- dores anglosajones han sabido utilizar desde hace varias décadas para construir una taxonomía que hoy ha sido aceptada por la antropología de la salud:

-Disease es lo que nosotros entendemos por enfermedad en su sentido recto, aquella de la que se ocupan los médicos, que hace referencia al mal funcionamiento del sistema fisiológico, su diagnóstico y su tratamiento (Lipson, 2000:21). El disease es la aprehensión biomédica de la enfermedad, fundada sobre un conocimiento objetivo de los sintomas físicos de la enfermedad, que implica por parte de la práctica médica de una ocultación o, por lo menos, una relegación al último plano de lo existencial y de lo social (Laplantine, 2992:19).

- Illness hace referencia a la experiencia cultural, interpersonal y personal (subjetiva) de la enfermedad. Se refiere a los problemas que la enfermedad produce al enfermo o incapacitado y a su familia (Eisemberg, 1977; Lipson, 2000:21). Posiblemente la palabra en castellano que mejor recoge esta definición es padecimiento (de hecho nosotros usamos harto frecuente el término paciente para referimos al enfermo-sujeto).

El padecimiento es más preocupante para la mayoría de las personas que la propia enfermedad, ya que ésta supone a menudo la iniciación en una terminología y una materia que le son extrañas y por lo tanto no tienen más remedio que delegar en los profesionales.

- Sickness se refiere a la dimensión simbólico-social de la enfermedad. Implica la presencia de ideologías en torno a la enfermedad, que llegan a generar estigmas (Martínez Hernáez, 172:61). Según Jean Benoist (1983:56) ha de utilizarse para designar el proceso de socialización del disease y del illness, permitiendo analizar los discursos, comportamientos y las prácticas sociales ante la enfermedad.

Un ejemplo muy claro lo tenemos en el caso del Sida, que supone un reto para la medicina en 
tanto no ha encontrado un remedio definitivo y por tanto es una enfermedad en constante proceso de indagación de sus causas y posibles terapias (disease), pero también es una enfermedad que debido a su peculiar curso clínico provoca un enorme sufrimiento a los pacientes como consecuencia del deterioro biológico que produce y las condiciones extremas en que se practican los cuidados (illness o padecimiento). A todo ello hay que añadir el malestar que provoca en el paciente el tener que enfrentarse a los estigmas que la sociedad se ha construido en torno a la enfermedad y que da lugar a respuestas de rechazo social y marginación (sickness).

Así pues desde este momento propongo una reformulación de los términos:

- enfermedad-enfermo, que han de referirse a la enfermedad-objeto (disease),

- padecimiento-paciente, para referirse a la enfermedad-sujeto (illness), y (sickness).

-malestar, reservado a la enfermedad-social

Si mi propuesta tiene éxito tendremos que aprender a usar con propiedad estos términos en el futuro (ver cuadro 1).

\section{Tiempo y espacio para los cuidados}

¿Pero qué utilidad puede tener para la enfermeria este modelo de segmentación del concepto de enfermedad? En realidad es determinante para configurar los diferentes espacios de cuidados en el proceso salud-enfermedad.

Pondré un ejemplo. Imaginemos el caso de un niño (Julián) que padece un transtorno neurológico degenerativo de origen desconocido. La enfermedad se mostrará en forma de un continuum que se va a desarrollar en tiempos y espacios diversos, en los que se alternarán la enfermedad, el padecimiento y el malestar (ver cuadro 2). Estos tiempos y espacios van a determinar el tipo de cuidados que Julián y su familia van a necesitar.
TIEMPO 1. Los primeros síntomas de la enfermedad se manifestaron en la escuela (espacio social) y se mostraron en forma de transtornos de la conducta (primero crisis de irritabilidad, luego mareos y colvulsiones repentinas), que provocaron inquietud a los alumnos compañeros de clase y a los profesores. La tensión entre los padres de Julián y los profesores, que recomiendan la no escolarización hasta resolver el problema, provocan una situación de malestar. A menos que exista un buen programa de salud escolar a nivel comunitario será muy difícil que una enfermera se encuentre con el problema y pueda actuar.

TIEMPO 2. Los padres llevan a Julián al médico, que recomienda su hospitalización para facilitar el proceso diagnóstico (espacio institucional médico). El niño es atendido por médicos y enfermeras que le hacen multitud de pruebas. Los médicos no se ponen de acuerdo sobre el origen del problema, Julián adquiere en ese momento el carácter de "caso raro" (interesante para la ciencia) y su presencia institucional se intensifica, es derivado a otros centros y conoce a nuevos especialistas que discuten entre ellos sobre su enfermedad. Los padres autorizan a los médicos a practicar tratamientos experimentales con su hijo, mientras Julián, que sufre la pérdida progresiva de algunas de sus facultades esenciales, va asimilando el ambiente institucional como algo inherente a su existencia (padecimiento). Finalmente se llega al diagnóstico: Julián sufre un fallo genético que provoca una degeneración del cerebro para el que hasta ese momento la medicina no puede dar una respuesta. Los médicos han encontrado limitaciones científicas y Julián adquiere el carácter de "caso imposible" (no interesante para la ciencia).

TIEMPO 3. Julián vuelve a su casa (espacio familiar) con un tratamiento paliativo prescrito por los médicos, que han pronosticado una muerte probable en un plazo de dos años. Julián adquiere desde ese momento la condición de desauciado (estig- 
ma), que le otorga su situación de invalidez tanto médica como social (malestar). Julián y su familia se enfrentan a las consecuencias de un proceso degenerativo severo que supone la adopción de habilidades cuidadoras y una implicación y apoyo permanentes. La familia entra en crisis porque ha de enfrentarse a un cambio en el sentido vital (padecimiento).

Mientras los padres de Julián aceptan el pronóstico médico lo normal es que reciban ayuda de su centro de salud, que realizará un control y seguimiento del problema en su calidad de enfermedad crónica y discapacitante. También podrán acogerse a programas institucionales de ayuda a domicilio e incluso podrán recibir apoyo informativo, emocional y material por parte de una asociación de ayuda mutua, de la que ellos como padres afectados pasarán a formar parte (la adscripción a la asociación de afectados es una manera de reafirmarse en el estigma).

TIEMPO 4. Los padres de Julián no aceptan el pronóstico (malestar), y compaginan el proceso de atención a su hijo (padecimiento) con un proceso de búsqueda de soluciones alternativas que, dependiendo de su marco cultural de referencia, les llevará a consultar nuevos especialistas (regreso al espacio institucional médico) y tal vez a utilizar terapias no convencionales (homeopatía, curanderos, centros religiosos, etc.), (espacio social).

En el continuum de Julián la implicación de la enfermera, el tipo e intensidad de los cuidados, va a variar en función del tiempo y de la alternancia en el espacio. Pero también va a variar dependiendo si dirige los cuidados al enfermo-objeto o al paciente-persona. La enfermería clínica tal como se ha entendido tradicionalmente dirige su atención con preferencia a controlar las variables que se asocian con la enfermedad (un ejemplo de ello son las tradicionales gráficas hospitalarias), mientras que dedica poco tiempo y esfuerzo a recomponer en un sentido amplio la vivencia del paciente durante su enfermedad, lo cual limita la eficacia de los cuidados y se contrapone muy a menudo a las expectativas del paciente.

El inscribir a la enfermería en un modelo que haga compatibles las necesidades del paciente con su contexto cultural (enfermería transcultural) supone desarrollar unas habilidades específicas, que tienen que ver fundamentalmente con la conversación y el lenguaje, a través de los cuales es revelada la cultura (Lipson, 2000:20), y con la observación y la comunicación no verbal (ocupación del espacio, la mirada, el estilo de conversación y tono de la voz, el tacto, concepto de tiempo, de modestia, etc.). También implica la adopción de instrumentos de valoración y de evaluación flexibles, pero sobre todo desarrollar en la enfermera el saludable hábito de la escritura.

La construcción de una antropología de los cuidados me parece importante porque nos ayuda a visualizar el padecimiento de la persona cuando se enfrenta a la enfermedad. Es una buena estrategia para dotar a los ideales humanizadores por los que todos luchamos como enfermeros de un marco de referencia teórica que posibilite la investigación y le de solidez científica. Pero también es una manera de escuchar a los que a menudo no tienen voz en el sistema hegemónico del que formamos parte: el paciente y su familia.

\section{BIBLIOGRAFÍA}

Amezcua M, Carricondo Guirao A (2000). Investigación cualitativa en España. Análisis de la producción bibliográfica en salud. Index Enfermería, 28-9:26-34

BENOIST J (1983). Quelques repères sur l'évolution récente de l'anthropologie de la maladie. Bull. D'Ethnomédecine, $9: 51-8$.

CAsares J (1942). Diccionario ideológico de la Lengua Española. Barcelona: Gustavo Gili.

EISEMberG L (1977). Disease and Illness. Culture, Medicine and Psychiatry, 1(1):9-23

FABREGA H (1975). The Need for an Ethnomedical Science. Science, 89:969-973.

LAPlantine F (1992). Anthropologie de la maladie. Paris: Éditions Payot. 
Leininger M. (1970). Nursing an Antropology: Two Worlds to Blend. New York: John Wiley.

-1978). Transcultural Nursing: Concepts, Theories and

Practices. New York: John Wiley \& Sons.

Lipson J.G. (2000). Cultura y cuidados de Enfermería. Index de

Enfermería, 28-9:19-25
Martinez Hernáez A. (1992). Eficacia simbólica, eficacia biológica. Hacia un nuevo modelo analítico y terapéutico en la asistencia sanitaria. Rev Rol Enferm, 172:61-7.

SOLAS O, UGALDE A, edit. (1995). Inmigración, salud y políticas sociales. Granada: Escuelas Andaluza de Salud Pública, Serie monografías n." 13 .
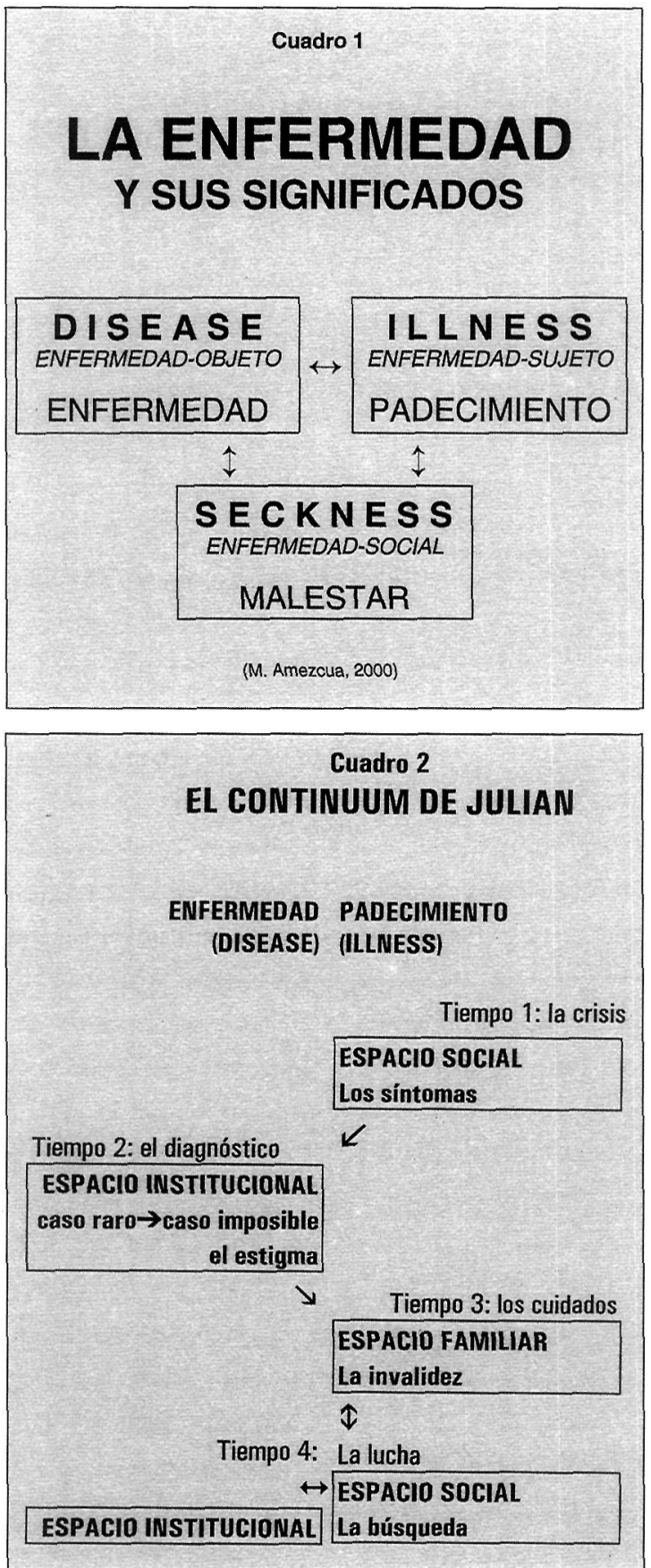